\title{
Origem e ramificação do tronco linguofacial em fetos de bufalinos sem raça definida (Bubalus bubalis bubalis)
}

\author{
Origin and branching of the linguofacial trunk in crossbred buffaloes fetuses (Bubalus bubalis bubalis) \\ Tathiana Ferguson Motheo ${ }^{1}$, Márcia Rita Fernandes Machadoํㅡㄹ \\ Wilson Machado de Souza ${ }^{2} \&$ Fabrício Singaretti de Oliveira ${ }^{3}$
}

\begin{abstract}
RESUMO
O tronco linguofacial é originado da artéria carótida comum externa, a qual pode ser utilizada para análise de pulso em ruminantes. Esta pesquisa foi desenvolvida com o intuito de melhor conhecer alguns aspectos deste tronco e seus ramos em fetos de bubalinos, uma vez que não existem informações precisas sobre esse assunto na literatura. Foram dissecadas vinte cabeças de fetos de bubalinos, injetadas com solução de látex e fixadas em solução de formol a 10\%. Na maioria dos casos, as artérias lingual e facial direita e esquerda apresentavam origem em tronco comum, denominado tronco linguofacial, proveniente da artéria carótida comum externa, na altura do ângulo formado pelo ramo e corpo da mandíbula. Em todas os casos, a artéria facial, após sua origem, contornava o músculo masseter e seguia direção dorso-rostral. Logo após sua origem, a artéria lingual emitia um ramo glandular e seguia trajeto rostral, dorsalmente ao músculo digástrico da mandíbula e sobre o músculo estiloglosso, aprofundando-se em direção ao ápice da língua e denominando-se artéria lingual profunda; a mesma também emitia a artéria sublingual que apresentava trajeto ao longo da borda dorsal do músculo geniohióide até a extremidade rostral da mandíbula. A origem do tronco linguofacial em bubalinos apresenta semelhanças ao descrito na literatura em relação aos bovinos, eqüinos e caprinos. A artéria facial apresentou trajeto similar ao de carnívoros, ruminantes e eqüinos e a artéria lingual trajeto semelhante ao de pequenos ruminantes, carnívoros e suínos.
\end{abstract}

Descritores: tronco linguofacial, anatomia, búfalo.

\section{ABSTRACT}

The linguofacial trunk arises from the external carotid common artery, which can be utilized for pulse analysis in ruminants. This researche was developed for purpose of better knowing some aspects of this trunk and its branches in buffaloes fetuses because there is no accurate information about it in literature. Twenty heads of buffaloes fetuses were dissected, injected with latex solution and set in a 10\% formaldehyde solution. In most of the cases, the right and left lingual and facial arteries arose from a common trunk, named linguofacial, originated from the external common artery, near the angle formed by the ramus and body of the jaw. In all the cases, the facial artery, after its origin, contoured the masseter muscle and followed a dorsorostral direction. Soon after its origin, the lingual artery gave origin to a glandular branch and kept a rostral path, dorsal to the digastric muscle of the jaw and over the styloglossus muscle, deepening to the tongue apex and named deep lingual artery; the same artery also gave origin to the sublingual artery, which paths trough the dorsal border of geniohyoid muscle up to the rostral tip of jaw. The origin of the linguofacial trunk in buffaloes resembles the present data in literature regarding bovine, equine and caprine. The facial artery presented path similar to the one of carnivorous, ruminant and equine and the lingual artery similar path to the one of small ruminants, carnivorous and pigs.

Key words: linguofacial trunk, anatomy, buffalo. 


\section{INTRODUÇÃO}

O tronco linguofacial é originado da artéria carótida comum externa, a qual pode ser utilizada para análise de pulso em ruminantes [8]. O pulso ainda pode ser detectado quando essa artéria é pressionada contra os processos transversos das vértebras [3].

As artérias lingual e facial também podem surgir separadamente, não caracterizando tronco. A primeira possui origem variável e muitas vezes surge separadamente da artéria carótida comum externa, emitindo um ou mais ramos para a glândula mandibular. A segunda é o ramo principal do tronco linguofacial e continua além da origem da artéria lingual, ascendendo a borda rostral do músculo masseter [5].

A artéria facial inicialmente apresenta trajeto medial à borda ventral da mandíbula, passando posteriormente à sua superfície lateral. Em carnívoros, ruminantes e eqüinos, a artéria facial segue a borda cranial do músculo masseter. Exceto em pequenos ruminantes, nos quais é ausente, a artéria facial origina ainda a artéria submentoniana que cursa medialmente à mandíbula, ventro-lateralmente ao músculo milohióideo em direção rostral, não chegando a atingir a mandíbula [8]. Apenas em ruminantes, a artéria lingual, durante seu trajeto até a língua, origina pequenos ramos glandulares que irrigam a glândula mandibular e a glândula parótida $[7,8]$.

Considerando-se que o conhecimento da ramificação vascular de uma região do organismo é fundamental para abordagens clínico-cirúrgicas precisas e que faltam informações sobre a morfologia detalhada dos búfalos, objetivou-se com este trabalho descrever a ramificação do tronco linguofacial nestes animais.

\section{MATERIAIS E MÉTODOS}

Para a realização desta pesquisa, foram utilizados vinte fetos de bufalinos sem raça definida (Bubalus bubalis bubalis), obtidos em frigoríficos da região de Taquaritinga, SP.

Após colheita, os fetos previamente congelados foram congelados e transferidos para o Laboratório de Anatomia Animal da Faculdade de Ciências Agrárias e Veterinárias (UNESP), Campus Jaboticabal, $\mathrm{SP}$, onde foram descongelados em água corrente, injetados com látex ${ }^{1}$ corado via aorta torácica, e fixados em solução aquosa de formol a $10 \%$ por período mínimo de 72 horas.

O tronco linguofacial, quando presente, foi mensurado mediante a utilização de um paquímetro digital $^{2}$.

\section{RESULTADOS}

\section{Origem das artérias lingual e facial direita e esquerda}

Em 70\% dos casos (14 peças), as artérias lingual e facial apresentavam, tanto no antímero direito quanto no esquerdo, origem em tronco comum, denominado tronco linguofacial, o qual era proveniente da artéria carótida comum externa, na altura do ângulo formado pelo ramo e corpo da mandíbula (Figura 1). Não houve caracterização do tronco linguofacial no antímero esquerdo em $15 \%$ dos casos (três peças). No direito, tal fato foi reportado em $10 \%$ dos casos (duas peças). Em apenas $5 \%$ dos casos (uma peça) não houve formação do referido tronco nos dois antímeros simultaneamente. Devido à ausência do tronco linguofacial nesses casos, as artérias lingual e facial apresentavam origem individual e eram provenientes da artéria carótida comum externa.

A média e o desvio padrão do comprimento do tronco linguofacial em bufalinos foram $0,45 \pm 0,23 \mathrm{~cm}$ no antímero direito e $0,48 \pm 0,24 \mathrm{~cm}$ no esquerdo. Os valores máximo e mínimo dos comprimentos foram, respectivamente, $0,21 \mathrm{~cm}$ e $1,10 \mathrm{~cm}$ no tronco linguofacial direito e $0,23 \mathrm{~cm}$ e $0,95 \mathrm{~cm}$ no esquerdo (Tabela 1).

\section{Trajeto e ramificação da artéria lingual}

Logo após sua origem, a artéria lingual emitia um ramo glandular e seguia trajeto rostral, dorsalmente ao músculo digástrico da mandíbula e sobre o músculo estiloglosso, aprofundando-se em direção ao ápice da língua, denominando-se, então artéria lingual profunda. Também emitia a artéria sublingual que possuía trajeto ao longo da borda dorsal do músculo geniohióide até a extremidade da mandíbula, não havendo diferenças entre os antímeros.

\section{Trajeto e ramificação da artéria facial}

Em todas as preparações, a artéria facial, após sua origem, contornava o músculo masseter e seguia direção dorso-rostral (Figura 2).

Inicialmente, da artéria facial, originavam-se as artérias labial mandibular superficial e labial mandibular profunda, as quais se dirigiam ao lábio inferior. Estas artérias tiveram origem comum em onze preparações (55\% dos casos), tanto no antímero esquerdo quanto no direito. A origem independente foi constatada em nove peças (45\% dos casos), nos dois antímeros.

A artéria facial emitia também, e direcionada ao lábio superior, a artéria labial maxilar, a qual origi- 


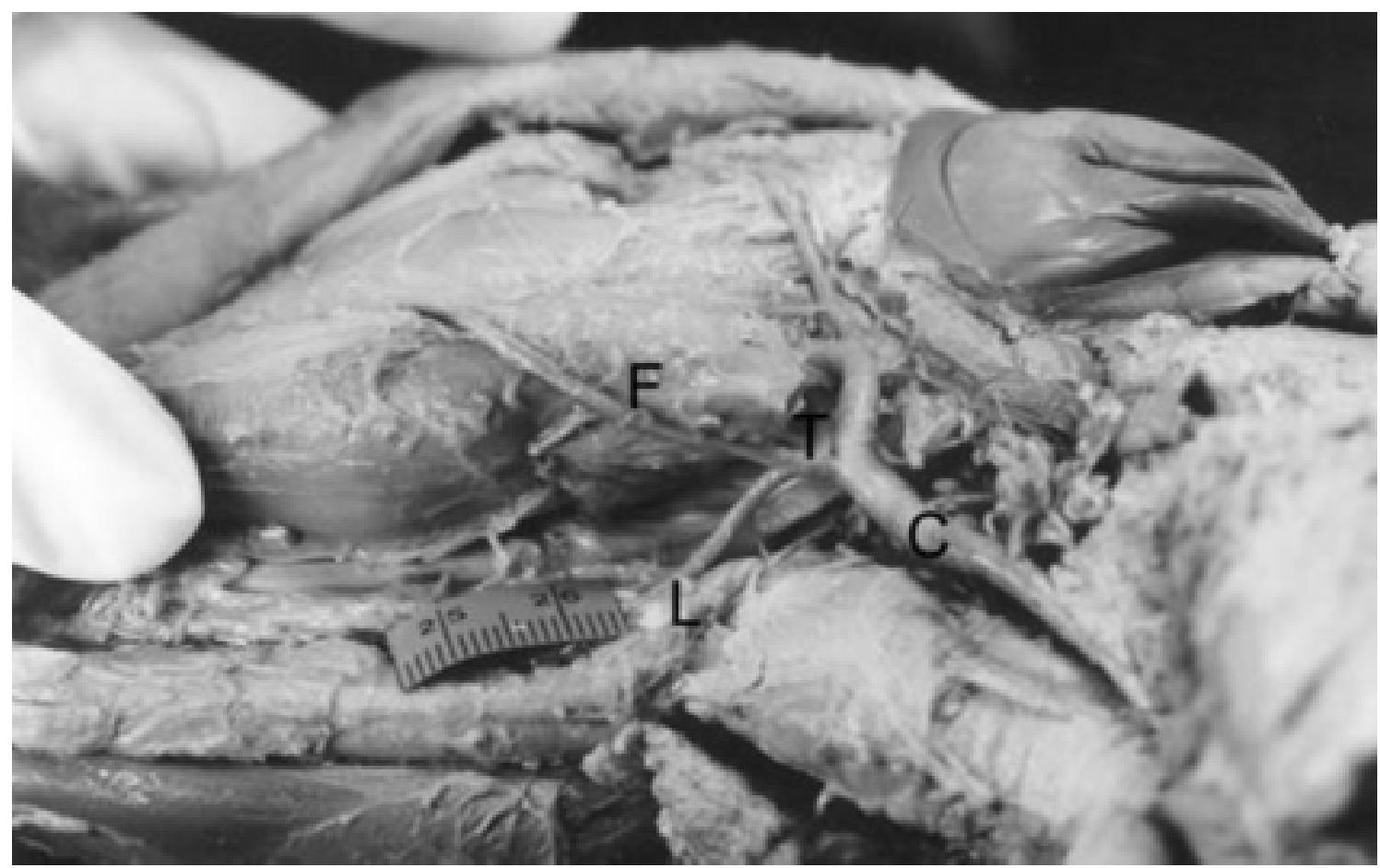

Figura 1. Parte da cabeça e pescoço (antímero esquerdo) de um feto bufalino, indicando a artéria carótida comum externa (C), e a origem, em tronco comum (T) e das artérias facial (F) e lingual (L).

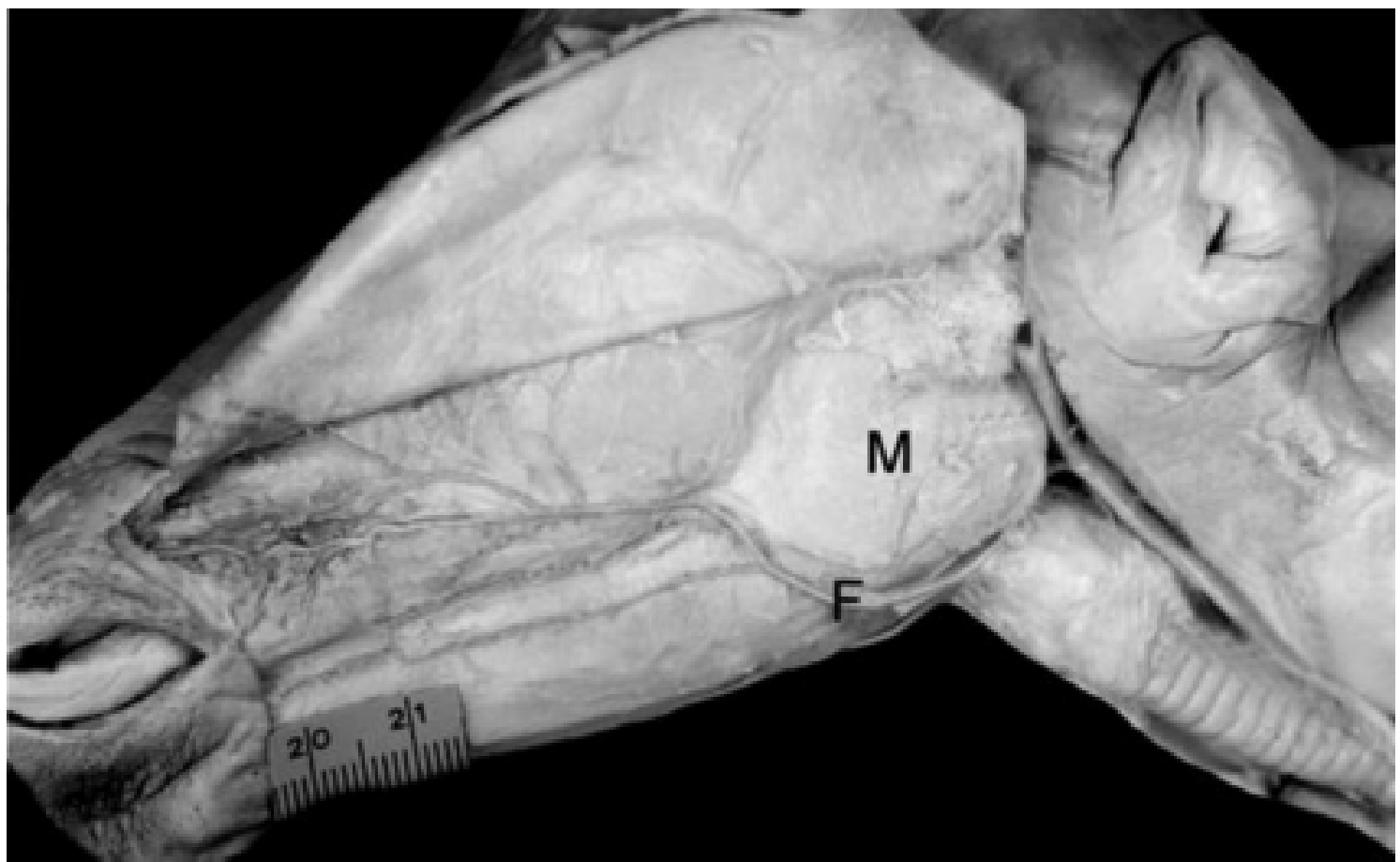

Figura 2. Trajeto da artéria facial esquerda $(\mathrm{F})$ na cabeça de um feto bufalino. Após sua origem, a mesma contorna o músculo masseter (M) e segue em direção dorso-rostral. 


\begin{tabular}{|c|c|c|}
\hline Número da amostra & $\begin{array}{l}\text { Comprimento do tronco } \\
\text { linguofacial direito }\end{array}$ & $\begin{array}{c}\text { Comprimento do tronco } \\
\text { linguofacial esquerdo }\end{array}$ \\
\hline 01 & 0,22 & 0,23 \\
\hline 02 & 0,25 & - \\
\hline 03 & 0,42 & 0,36 \\
\hline 04 & 0,50 & 0,60 \\
\hline 05 & 0,45 & 0,41 \\
\hline 06 & 0,62 & 0,80 \\
\hline 07 & 0,69 & 0,84 \\
\hline 08 & 0,21 & - \\
\hline 09 & 0,70 & 0,81 \\
\hline 10 & 0,29 & 0,31 \\
\hline 11 & 0,31 & 0,25 \\
\hline 12 & - & - \\
\hline 13 & 0,30 & 0,29 \\
\hline 14 & 0,45 & - \\
\hline 15 & 0,22 & 0,39 \\
\hline 16 & - & 0,35 \\
\hline 17 & 0,46 & 0,41 \\
\hline 18 & - & 0,32 \\
\hline 19 & 1,10 & 0,95 \\
\hline 20 & 0,41 & 0,41 \\
\hline Média & 0,45 & 0,48 \\
\hline Desvio Padrão & 0,23 & 0,24 \\
\hline
\end{tabular}

nava a artéria angular da boca, além de duas artérias laterais nasais rostrais.

\section{DISCUSSÃO}

A origem das artérias lingual e facial em tronco comum, denominado linguofacial, a partir da artéria carótida comum externa descrita em bovinos e eqüinos [3,7,8] e em caprinos sem raça definida [9] foi observada em dezessete peças no antímero direito e em dezesseis no esquerdo. A origem individual dessas artérias, não caracterizando tronco, também foi descrita nos bubalinos, similarmente ao descrito para bovinos, eqüinos e caprinos sem raça definida [3,7-9].

A artéria facial dos bufalinos apresentou trajeto semelhante àquele descrito em carnívoros, rumi- nantes e eqüinos, contornando o músculo masseter e seguindo direção dorso-rostral [5,8]. Não foi constatada a presença da artéria submentoniana, observada em pequenos ruminantes [2,5].

Nos bufalinos, os ramos para as glândulas salivares parótida e mandibular originaram-se da artéria facial, da mesma forma que em caprinos negros de Bengala [6] e diferentemente dos caprinos sem raça definida, cujos ramos originados da artéria facial irrigavam ou dirigiam-se apenas para a glândula salivar mandibular [9].

Assim como em carnívoros, bovinos e eqüinos, também foi constatada origem dorsal para a artéria labial maxilar e ventral para a artéria labial mandibular em bufalinos. Similarmente ao descrito para os rumi- 
nantes, a artéria labial mandibular apresentou-se dupla, dividindo-se em um ramo delgado, a artéria labial mandibular superficial, e um outro espesso, a artéria labial mandibular profunda [2,7-9].

Similarmente ao descrito nos ruminantes e suínos, as artérias lateral nasal rostral, angular da boca e angular do olho originam-se do ramo terminal da artéria facial dos bufalinos [7].

A artéria lingual originou-se a partir da artéria carótida comum externa, de forma individual em relação à artéria facial ou em tronco comum a esta, de modo semelhante ao descrito para pequenos ruminantes, carnívoros e suínos [2,5,7].

Em relação ao trajeto da artéria lingual, esta se dirige rostroventralmente e penetra na língua pelo aspecto medial do músculo mioglosso, emitindo os ramos glandulares mandibulares, similarmente ao descrito em caprinos negros de Bengala [6]. Emite também os ramos perihioídeos, a artéria profunda da língua e ramos dorsais linguais, como reportado para carnívoros, suínos, pequenos ruminantes e ruminantes [5,7-9].

\section{CONCLUSÕES}

A origem do tronco linguofacial em bubalinos apresenta semelhanças ao descrito na literatura em relação aos bovinos, eqüinos e caprinos. A artéria facial apresentou trajeto similar ao de carnívoros, ruminantes e eqüinos e a artéria lingual trajeto semelhante ao de pequenos ruminantes, carnívoros e suínos.

\section{NOTAS INFORMATIVAS}

${ }^{1}$ Neoprene $650^{\circledR}$, Du Pont do Brasil S.A., SP/Brasil.

${ }^{2}$ Tesla ${ }^{\circledR}$, Genebra, Suíça.

Agradecimentos. Projeto financiado pela FAPESP (Fundação de Amparo à Pesquisa do Estado de São Paulo), processo de iniciação científica número 01/05918-9.

\section{REFERÊNCIAS}

1 Barnwal A.K. \& Sinha R.D. 1983. Anatomical studies on the blood supply of the salivary glands of buffalo. Indian journal of Animal Sciences. 53: 503-507.

2 Bruni A.C. \& Zimmerl U. 1951. Anatomia degli animali domestici. 2nd edn. Milão: Francesco Vallardi, pp.322-325.

3 Dyce J.M., Sack W.O. \& Wensing C.I.G. 1997. Tratado de anatomia veterinária. Rio de Janeiro: Guanabara Koogan, pp.508-509.

4 Godinho H.P., Cardoso F.M. \& Nascimento J.F. 1985. Anatomia dos ruminantes domésticos. (Apostila). Belo Horizonte: UFMG. 337p.

5 Goshal N.G. 1986. Sistema Circulatório. In: Getty R. (Ed). Anatomia dos animais domésticos. 5.ed. v.1. Rio de Janeiro: Interamericana, pp.874-907.

6 Hossain M.I. 1975. The arterial supply to the salivary gland of Black Bengal goat. Indian Veterinary Journal. 52: 699-702.

7 Nickel R., Schummer A., Seiferle \& Sack W.O. 1981. The circulatory system, the skin, and the cutaneous organs of the domestic mammals. Berlin: Verlag Paul Parey, pp.102-111.

8 Scharze E. \& Schröder L. 1972. Compendio de anatomia veterinaria: aparato circulatório y piel. v.3 Zaragoza: Acribia, p.31.

9 Silva F.O.L., Bombonato P.P., Rodrigues C.A., Severino R.S. \& Santos A.L.Q. 1988. Presence of the facial artery in goats. Revista da Faculdade de Medicina Veterinaria e Zootecnia da Universidade de São Paulo. 25: 309-315. 